\title{
Effect of LMC on the Galactic Warp
}

\author{
Toshio Tsuchiya \\ Astronomisches Rechen-Institut, Mönchhofstraße 12-14, 69120 \\ Heidelberg, Germany
}

\begin{abstract}
Multi-scale interaction between LMC, the Galactic halo, and the disk is examined by N-body simulations. The Galactic models are constructed most realistically to satisfy available observational constraints. LMC is orbiting in a ellipse with pericentric radius of $50 \mathrm{kpc}$, and the Galactic halo is assumed to be well beyond the orbit. By using hybrid algorithm I have succeeded to follow the evolution with million particles. The orbiting satellite excites density enhancement as a wake, and the wake exerts a tidal force on the disk. Through this multi-scale interaction, an integral warp is excited. Its amplitude is, however, a few 100pc, and impossible to explain the observed Galactic warp.
\end{abstract}

\section{Introduction}

Large fraction of disk galaxies shows bending of disks in an integral shape, which is called warp, so that the warp must be a quite basic and possibly very important phenomenon in the formation and evolution of the galaxies. The Milky Way also has prominent warp, but even for our system, the origin of the warp is still unclear. A possible mechanism is interaction with satellites. The LMC seems the most suitable because of its large mass $\left(\sim 10^{10} M_{\odot}\right)$, and position (its torque is the right sense to create the warp). Though it is known that the direct torque from the LMC is not strong to excite observed amplitude, Weinberg (1998) suggested that this problem might be cured by considering the following processes. (1)A satellite (LMC) is orbiting in a extended halo. (2) Density waves (wakes) are excited owing to the satellite's motion. (3) A resonant wake appears nearly half distance to the satellite, and its density excess is about the same mass as the satellite. (4) The tidal torque from the wake bend the galactic disk, which is comparable to the observed one.

It is necessary to examine this scenario by fully self-consistent $N$-body simulations with very large number of particles to ensure enough resolution to cover such multi-scale dynamics.

\section{Numerical Simulations}

Our numerical code employs a hybrid algorithm, called SCF-TREE (Vine \& Sigurdsson 1998). SCF (Self-Consistent Field method) is an expansion method, in which density and potential are simultaneously expanded by a bi-orthogonal 

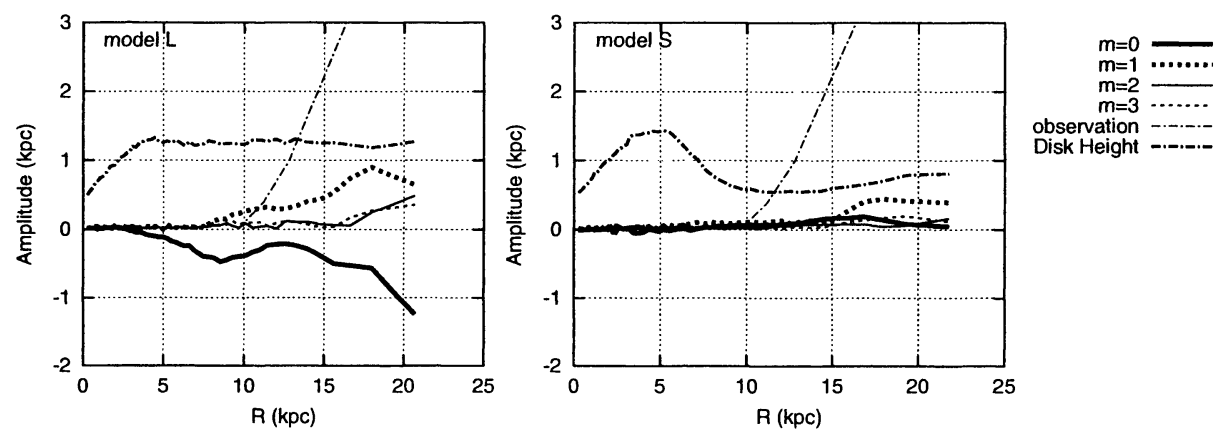

Figure 1. Amplitudes of harmonic warp modes

basis set. The angular dependences are expanded by the usual spherical harmonics, and radial functions are based on the Hernquist's profile and the Gegenbauer polynomials. The disk and bulge are dealed by TREE, and the halo is treated by SCF part. Each part contains 524,288 particles, so that the system consists of more than 1 million particles in total.

For numerical construction of equilibrium Galaxy models, we used Kuijken \& Dubinski (1995)'s models, which include a halo, bulge, and disk. The bulge has the distribution function same as the King model. The halo is Evans's extension of the King model, which allows $L_{z}$ dependence. The disk has an exponential surface density profile and nearly isothermal vertical distribution. We have chosen the model controlling parameters so that the models satisfy available observational constraints for the Milky Way. The models, however, still allow free choice of the halo extent. Therefore we have made 2 models: the model $\mathbf{L}$, which has very large extended halo, and the model $\mathbf{S}$, which has minimum halo mass. The LMC is modeled by a extended single particle, which has the Hernquist profile, with mass $M_{\mathrm{LMC}}=10^{10} M_{\odot}$.

Figure 1 shows the harmonic amplitude of disk warping modes after $6 \mathrm{Gyr}$ evolution of the Milky Way-LMC system. The mode of $m=1$ corresponds to the integral warp. The thin dotted-dashed curves show the observed warp amplitude. The model $\mathrm{L}$ yields larger warp amplitude than model S. This results indicates that the halo does play important roll on excitation of warps. From both figures, however, it is clear that the disk warping obtained by my simulations is much smaller than the observed one, irrespective of the halo distribution. Though more parametric study is necessary, at least conventional Milky Way models are hard to produce a warp as big as the observed one.

\section{References}

Kuijken, K., \& Dubinski, J. 1995, MNRAS277, 1341

Vine, S., \& Sigurdsson, S. 1998, MNRAS295, 475

Weinberg, M. D. 1998, MNRAS, 299,499 